(C) 2019 IEEE. Personal use of this material is permitted. Permission from IEEE must be obtained for all other uses, in any current or future media, including reprinting/republishing this material for advertising or promotional purposes, creating new collective works, for resale or redistribution to servers or lists, or reuse of any copyrighted component of this work in other works. 


\title{
Acoustic Sensor Networks and Mobile Robotics for Sound Source Localization
}

\author{
Linh Nguyen $^{1}$ and Jaime Valls Miro ${ }^{1}$
}

\begin{abstract}
Localizing a sound source is a fundamental but still challenging issue in many applications, where sound information is gathered by static and local microphone sensors. Therefore, this work proposes a new system by exploiting advances in sensor networks and robotics to more accurately address the problem of sound source localization. By the use of the network infrastructure, acoustic sensors are more efficient to spatially monitor acoustical phenomena. Furthermore, a mobile robot is proposed to carry an extra microphone array in order to collect more acoustic signals when it travels around the environment. Driving the robot is guided by the need to increase the quality of the data gathered by the static acoustic sensors, which leads to better probabilistic fusion of all the information gained, so that an increasingly accurate map of the sound source can be built. The proposed system has been validated in a real-life environment, where the obtained results are highly promising.
\end{abstract}

\section{INTRODUCTION}

Recently, sound source localization has been attracted in many nowadays-concerned researchs comprising automatically steering a camera to the direction of a speaker in a teleconferencing room [1]-[4], separating multiple speaker speeches [5], detecting a source in an environment where it requires privacy preserve or has poor lighting conditions and occlusions [6]-[8], search and rescure [9], [10] and mapping a 3D source in autonomous robotic systems [11], [12]. The work hereby proposed has been motivated by aged care applications such as robot audition for aged care services, where it needs to add machine intelligence and smart automation to the way society deals with the substantial challenges that an increasingly ageing population presents. As shown in Fig. 2, given advantages of modern technology, robots may be well liked to assisting the elderly and frail in their daily activities. Nevertheless, in the cases where there are challenging lighting conditions or vision sensors are not allowed due to privacy, how the robot could interact with their masters if it cannot localize them? Therefore, this work proposes to exploit advances in sensor networks, robotics and acoustic research to locate a sound source (people are considered as sound sources when they speak). In the aged care application, for instance, it is to assist the frail and elderly maintain their quality of life by leading safe independent lives in as much as possible, and doing so while respecting users' privacy rights. The main objective of the research work resides in mapping a likely location of a sound source based on their acoustic footprint.

\footnotetext{
${ }^{1}$ Centre for Autonomous Systems, University of Technology, Sydney, New South Wales 2007, Australia vanlinh.nguyen@uts . edu . au and jaime.vallsmiro@uts.edu.au
}

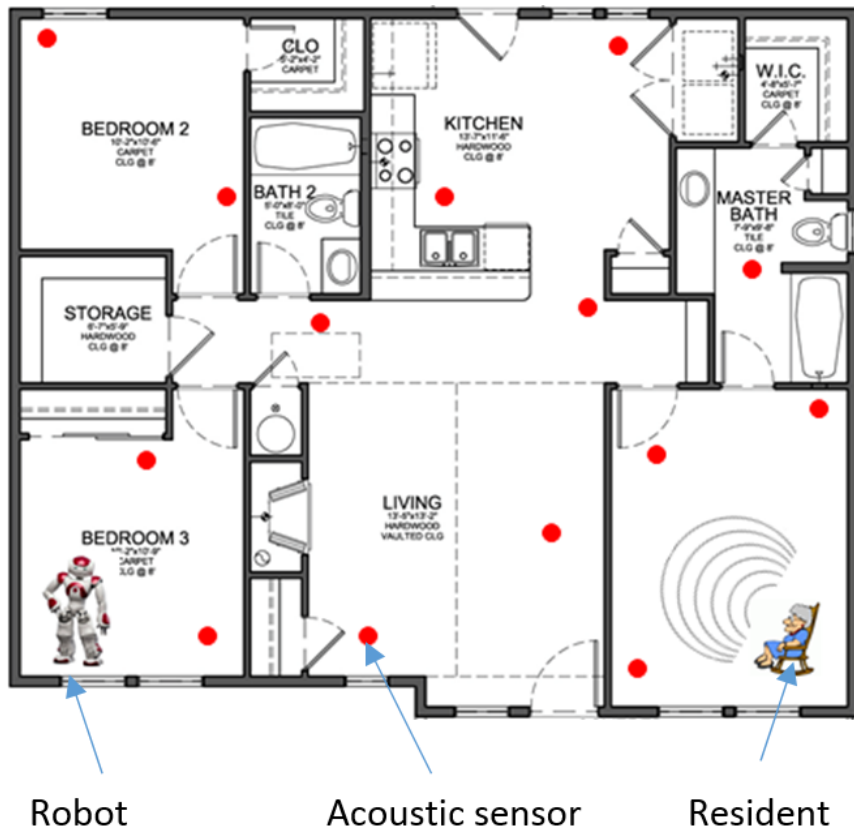

Fig. 1: Acoustic sensor networks and mobile robotics for health cares.

Basically, there are two categories of the methods proposed to find a source given its sound signals captured by microphone sensors. The first type is a direct approach [13] that aims to maximize the steered response power of the output of a delay and sum beamformer, which delays the outputs of the microphone sensors and adds them together so that it can reinforce the signal to noise ratio. In contrast, localizing a sound source in the indirect method requires two separate steps [14]. Time difference of arrival (TDOA) [15] is firstly estimated from the generalized cross-correlation peaks [16] of a microphone signal pair. Then the source location, given the correspondingly estimated TDOAs, can be ascertained by optimally addressing an optimality criteria [17] such as the hypercone fitting problem [18]. Nevertheless, results obtained the indirect method are quite sensitive to the presence of noises and reflections/refractions [19].

To acquire sound signals, a network of acoustic sensors introduces a new paradigm, where each sensor node encompasses microphones or microphone arrays [14], [20]. Sensor networks consist of spatially distributed autonomous devices using sensors to cooperatively monitor physical or environmental conditions [21]-[32], sound in this case, at different locations to better predict the distribution of the 
underlying phenomenon of interest. In employing acoustic sensors networks over e.g. video cameras the data stream can preserve anonymity, becomes impervious to challenging lighting conditions and can deal more efficiently with occlusions. Moreover, due to challenges in the acoustic signals, in that as distance from a sound source increases their signalto-noise ratio decreases rapidly, reflections of sound on the environment make sound source location particularly challenging. While only bearing to sound sources can be directly inferred from measurements with a single microphone array, the proposition of using a network of acoustic sensors so that additional spatial measurements of likely sound sources can be gathered and fused together to get more robust predictions of the sound sources. On the other hand, one of the key drawbacks of WSN is precisely their static nature. Thus, a mobile robot with an additional microphone array is employed so that active sound observations can be strategically recorded at locations of choice. These will be guided by the need to increase the quality of the information gathered by the static acoustic sensors, and that of the probabilistic fusion process itself, so that increasingly accurate maps of sound sources can be built. The proposed system has been extensively evaluated in the realistic experiments, where the sound source was algorithmically localized by the steered response power - phase transform approach.

The remaining of the paper is arranged as follows. Section II introduces a method to localize a sound source while the proposed system is presented in Section III. Section IV descries how the experiments were conducted and analyses the results before conclusions are drawn in Section V.

\section{Sound Source Localization ApproACH}

In this section, we will discuss how a sound source is localized based on the acoustic data collected by a network of the acoustic microphone sensors fixed at static locations or carried by a mobile robot. It first presents a reverberation model of the acoustic signals. The microphone signals are then fed into the delay-and-sum beamformer to compute the locations of a source.

\section{A. Signal Model}

Consider a network of $M$ microphone sensors that are deployed arbitrarily in a reverberation environment. A signal acquired by the $m^{t h}$ microphone $(m=1, \cdots, M)$ at time $t$ can be presented by a reverberation model [8], [33] as follows,

$$
a_{m}(t)=h_{m}(t) \odot s(t)+\epsilon_{m}(t)
$$

where $\odot$ denotes the linear convolution operator, $h_{m}(t)$ is the complete room impulse response from the sound source to the $m^{t h}$ microphone sensor, $s(t)$ is the sound source signal and $\epsilon(t)$ is the additive noise. Normally, $\epsilon(t)$ is assumed to be uncorrelated with $s(t)$ and a noise at another sensor.

In this work, all sound signals collected by the microphone sensors are processed in a frame to frame basis. Hence, samples of a frame with a length of $L_{f}$ at the $m^{t h}$ microphone and a discrete time $k$ can be specified by

$\mathbf{a}_{m}(k)=\left[a_{m}\left(k L_{f}\right), a_{m}\left(k L_{f}+1\right), \cdots, a_{m}\left(k L_{f}+L_{f}-1\right)\right]$.

\section{B. Steered Response Power - Phase Transform Approach}

Lets imagine that the sound signals $a_{m}(t)$ collected by the acoustic sensors are delayed, filtered and noise corrupted versions of the sound source signal $s(t)$. Hence, the delayand-sum beamformer [16] aims to align and add all the sound signals together to prevent the signals to be modified from a spatial location. Mathematically, the delay-and-sum beamforming can be presented by

$$
b(t, \tau)=\sum_{m=1}^{M} a_{m}\left(t-\left(\tau_{m}-\tau_{0}\right)\right)
$$

where $\tau_{m}$ is the time delay from the sound source to the sensor $m$ and $\tau_{0}$ is the minimum of all the time delays, $\tau=$ $\left(\tau_{1}, \tau_{2}, \cdots, \tau_{M}\right)$. Due to noise and reverberation, an adaptive filter can be employed to filter the acoustic signals before they are time aligned and summed. In the frequency domain, output of a filter-and-sum beamformer can be written by

$$
B(\omega, \tau)=\sum_{m=1}^{M} \Phi_{m}(\omega) A_{m}(\omega) e^{j \omega\left(\tau_{m}-\tau_{0}\right)}
$$

where $A_{m}(\omega)$ and $\Phi_{m}(\omega)$ are the Fourier transforms of the sound signal $a_{m}(t)$ and the filter, respectively.

If we steer the beamformer over a region covering a possible sound source, then its steered response power (SRP) can be computed by

$$
\begin{aligned}
P(\tau) & =\int_{-\infty}^{\infty} B B^{*} d \omega \\
& =\int_{-\infty}^{\infty} \sum_{n=1}^{M} \sum_{m=1}^{M} \Psi(\omega) A_{n}(\omega) A_{m}^{*}(\omega) e^{j \omega \Delta_{m n}} d \omega
\end{aligned}
$$

where $\Psi(\omega)=\Phi_{n}(\omega) \Phi_{m}^{*}(\omega), \Delta_{m n}=\tau_{m}-\tau_{n}$ and ${ }^{*}$ is the complex conjugate operator. If the weighting factor $\Psi(\omega)$ is defined by the well-known phase transform (PHAT) [34]

$$
\Psi(\omega)=\frac{1}{\left|A_{n}(\omega) A_{m}^{*}(\omega)\right|},
$$

then the PHAT based SRP can be rewritten by

$$
P(\tau)=\int_{-\infty}^{\infty} \sum_{n=1}^{M} \sum_{m=1}^{M} \frac{A_{n}(\omega) A_{m}^{*}(\omega)}{\left|A_{n}(\omega) A_{m}^{*}(\omega)\right|} e^{j \omega \Delta_{m n}} d \omega .
$$

The SRP-PHAT algorithm is then employed to find a location of a sound source by searching all possible locations in a pre-defined region. At each possible location, the power of the steered response $P(\tau)$ is computed. Therefore, the sound source location $l_{s}$ is a solution of an optimization problem as follows,

$$
l_{s}=\underset{\text { All possible locations } l}{\operatorname{argmax}} P(\tau) .
$$

For the purpose of practicality, the searching region is discretized into discrete locations. The finer the discretization is, the more accurately the source location is found. 


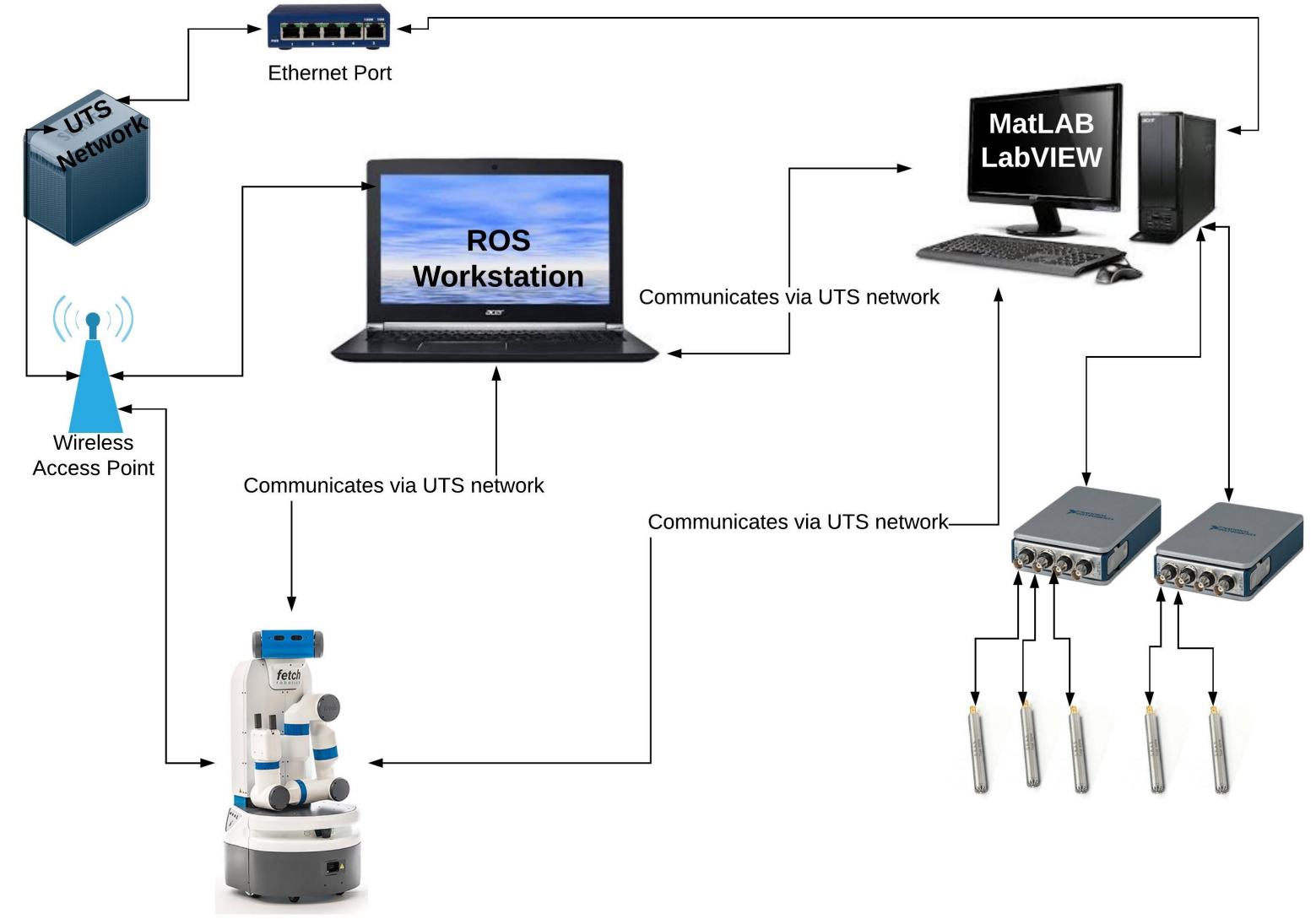

Fig. 2: The overall system of a sensor network and a mobile robot for sound source localization.

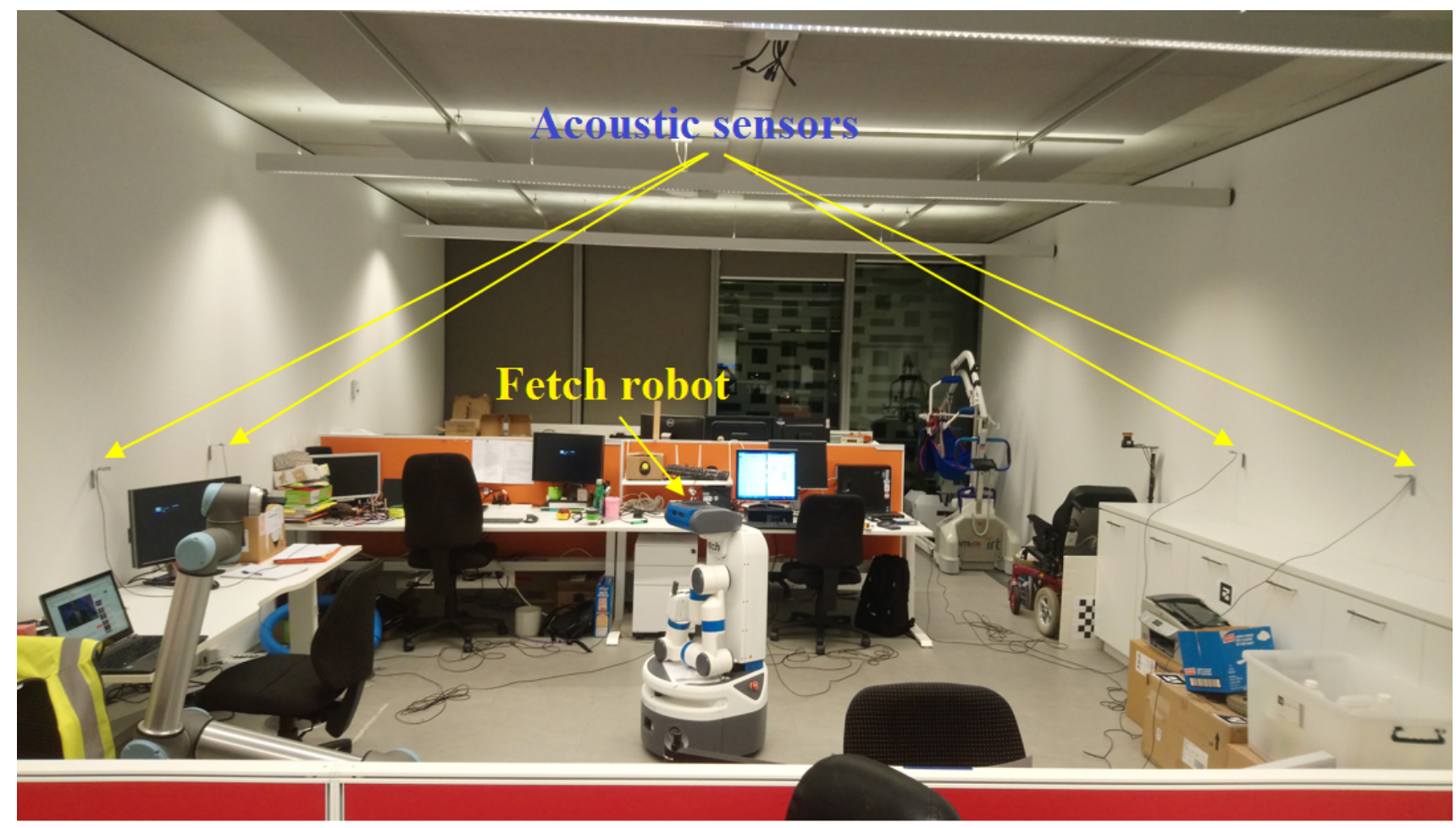

Fig. 3: Experimental set-up using the Fetch robot and the G.R.A.S. type 40PH free-field acoustic sensors. 


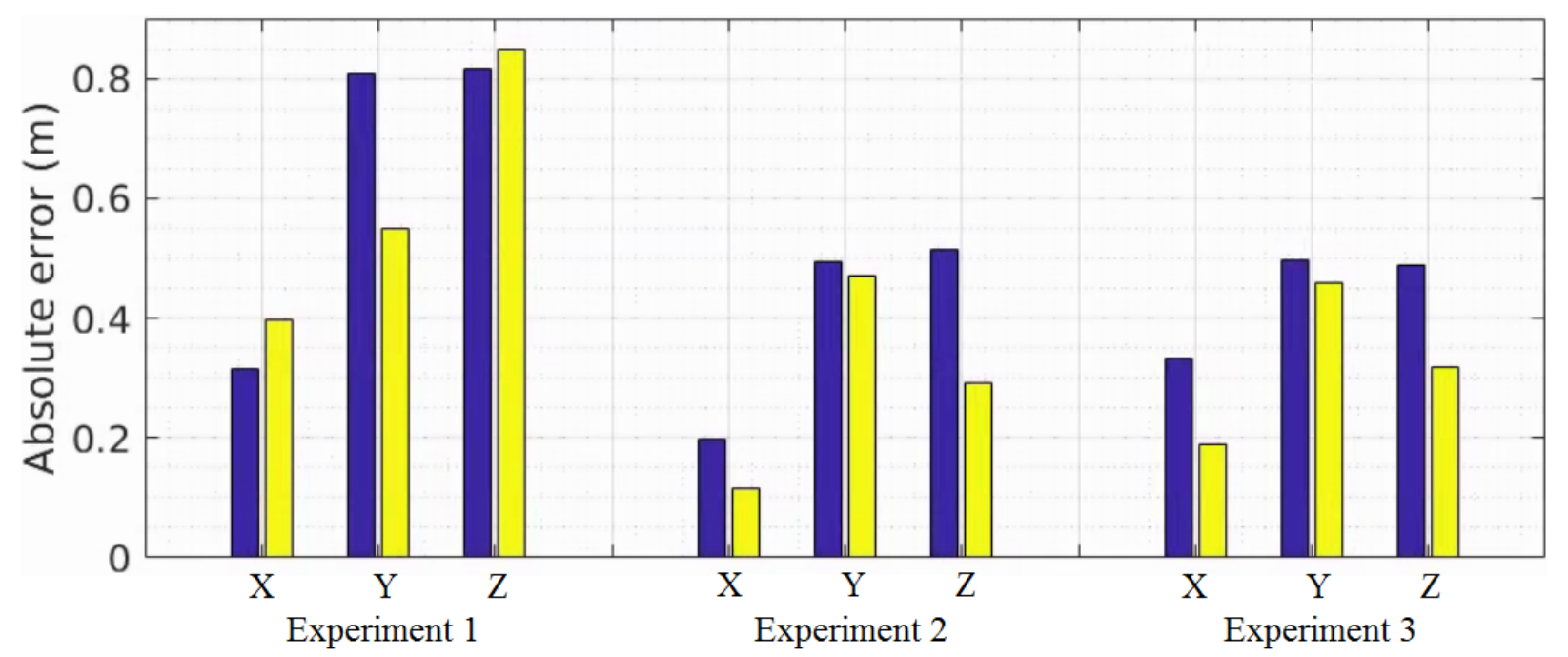

Fig. 4: Experimental absolute errors in three directions: Blue bars are obtained by the static network while yellow bars are obtained by the network and a mobile robot.

\section{OVERALL SYSTEM}

The overall system of an acoustic sensor network and a mobile robot for sound source localization is demonstrated in Fig. 2. It comprises a network of acoustic microphone sensors, where each of them is connected to a computer via a data acquisition module. The data gathered by the acoustic sensors is then wirelessly or wiredly transmitted to a central station for further processing. In addition, a mobile robot is controlled by an algorithm programmed on Robot Operating System (ROS) installed on a computer, which can be treated as the central station. In this work, for the purpose of demonstration, we implemented the system on the equipment as presented in the following.

\section{A. Robot Navigation}

In the work, we employed the Fetch robot [35] to conduct experiments. The Fetch robot is a mobile manipulator that is a standard platform for service robot applications. In order to drive the robot to navigate through environment, it exploits the ROS navigation stack and the robot's sensors including a 2-dimensional scanning laser rangefinder, a camera and an inertial measurement unit.

1) Robot localization: At every moving step, the robot needs to be localized on a pre-built map by the use of the adaptive Monte Carlo localization approach. This probabilistic localization method employs the particle filter to track a pose of the robot on a known map, based on the data collected by the laser sensor. In the proposed system, since the robot carries an acoustic sensor to collect sound signals, at every moving step, the robot's location is recorded for the sound source localization analysis.

\section{B. Acoustic Sensors and Data Acquisition}

The experiment equipment also comprises the G.R.A.S. type 40PH free-field microphones and the National Instruments (NI) modules of the compact data acquisition cDAQ-
9171 and the ADCs NI 9234. The sound signals are then recorded by the NI LabVIEW $\mathrm{R}$.

\section{Communication}

All the acoustic sensors are synchronously connected to the computers while the Fetch robot possesses an external Ethernet based communication capability that allows it to wirelessly communicate with other equipment. Therefore, a local area network can be set up to connect all the elements in the proposed system.

On the other hand, there are three different software exploited in the proposed system. While the NI LabVIEW (R)is required to run the data acquisition modules and ROS is

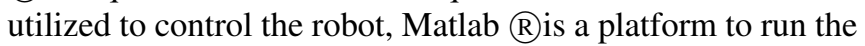
sound source localization algorithm. It is noted that in order to localize a sound source all the sound signals collected by the acoustic sensors are required to be synchronous and the sensor positions are available. Since the robot carries the microphone to travel around, its locations are a part of requirement for the sound source localization algorithm. Hence, to operate all the elements simultaneously, it is proposed Matlab as a primary platform, where it can call ROS through the robotics toolbox and LabVIEW through the ActiveX.

\section{EXPERIMENTS AND RESULTS}

\section{A. Experiments}

To study the performances of the proposed system in reallife scenarios, we conducted the experiments in the realistic noisy and reverberant conditions in the campus of University of Technology Sydney, Australia. The experimented environments are daily working laboratories where staff and students were walking, talking, discussing and doing their own works. Therefore, there have noises coming from many sources such as human activities, door opening and closing, ventilation fans, air conditioners and research/study equipments. There also exist polluted noises from the traffic roads nearby. All 
these presented noise elements were naturally captured by the microphones during the recordings, which leads to the very noisy measurements in our experiments. The reverberation times and SNRs in both realistic environments were unknown at the time of the experiments.

The experiment equipment comprised five the G.R.A.S. type 40PH free-field microphones and two National Instruments modules of the compact data acquisition cDAQ-9171 and the ADCs NI 9234. Dimensions of the laboratory room are about $12.0 \mathrm{~m} \times 5.8 \mathrm{~m} \times 3.0 \mathrm{~m}$. Four acoustic sensors were located against the walls and one microphone sensor was embedded on the Fetch robot as shown in Fig. 3. The sound source was a mobile phone continuously playing an audio recording of a speech utterance by a female speaker. The sound signals were then recorded by the NI LabVIEW 2014@. All the measurements were sampled at frequency of $16 \mathrm{kHz}$ with a resolution of 24-bits. The robot intermittently navigated through the room. It repeatedly travelled two seconds and stopped for five seconds. At each stop of the robot, all the microphone sensors including the static sensors and the dynamic sensor embedded on the robot synchronously recorded the five second sound signals. The robot stopped at five locations in each experiment. It is noticed that the locations of the robot when it stopped were identified by the robot's sensors. These information were utilized to compute the location of the dynamic sensor, which is required for the SRP-PHAT algorithm. All the recorded data was then transmitted to the central station for analysing the sound source location. There are three differently set-up experiments conducted in this work.

1) Experiment 1: All the acoustic sensors were located at the same height.

2) Experiment 2: Four static acoustic sensors were located at the same height, but the microphone on the robot was lower.

3) Experiment 3: All the acoustic sensors were located at random heights.

It is noted that in all the experiments, the sound source was deployed at a random location. Moreover, the robot was travelling on the randomly generated paths. And it is assumed that the sound propagation speed was set to $343 \mathrm{~m} / \mathrm{s}$. In the signal processing procedure, each microphone recording was split into frames with a length of 2048 samples and overlap of $50 \%$.

\section{B. Results}

For the purpose of comparisons, in the work we also conducted the static experiments, where all the microphones were fixed at specific locations. In these experiments, if the first four microphones were located at as the similar positions as they were set in the dynamic experiments as delineated in the section IV-A, the fifth acoustic sensor, which was embedded on the robot in the dynamic experiments, was deployed in the middle of the room at the height similar to the robot's height. Period of time to collect the data in the static experiments was as the same as that in the dynamic experiments. All the recorded sound signals were also sent to the central station to work out the location of the sound source by the use of the SRP-PHAT algorithm.

The results are demonstrated in Fig. 4 and Table I, where we computed absolute errors in three directions and computed root mean square errors (RMSEs) between the estimated locations and the real locations of the sound sources in all the experiments, respectively. In Fig. 4, while the yellow bars present the errors obtained by the dynamic experiments, the blue bars show those obtained by the static experiments. It can be clearly seen that in the experiments 2 and 3 , the absolute errors obtained by the proposed system are smaller than those obtained by the static network in all the directions. Nonetheless, in the experiment 1 , not only are the errors high but in the dynamic network they are worse in $X$ and $Z$ as compared to those in the static network. This is due to the fact that in the experiment 1 the source was deployed very close to a ventilation fan, which is very noisy.

However, in Table I, as expected, the proposed system outperforms the static network in terms of estimating the source locations. Due to using the robot movements, information collected by the dynamic network is richer than that of the static network, which eventually leads to the smaller RMSEs.

TABLE I: RMSEs FOR SOUND SOURCE LOCALIZATION

\begin{tabular}{|c|c|c|}
\hline & \multicolumn{2}{|c|}{ RMSEs (m) } \\
\hline & Static network & Network with robot \\
\hline Experiment 1 & 1.19 & 1.09 \\
\hline Experiment 2 & 0.74 & 0.56 \\
\hline Experiment 3 & 0.77 & 0.59 \\
\hline
\end{tabular}

Note that all the processing procedures were carried out on the platform of Matlab 2016 $\mathrm{R}$ on a 64-bit PC with computational capability of Intel(R) Core(TM) i5-6500 CPU (a) $3.20 \mathrm{GHz}$ and memory of $8.00 \mathrm{~GB}$. The running time of the algorithm in all the experiments is summarized in Table II.

TABLE II: COMPUTING TIME

\begin{tabular}{|c|c|c|}
\hline & \multicolumn{2}{|c|}{ Computing time (s) } \\
\hline & Static network & Network with robot \\
\hline Experiment 1 & 19.43 & 21.36 \\
\hline Experiment 2 & 19.95 & 22.01 \\
\hline Experiment 3 & 18.91 & 20.98 \\
\hline
\end{tabular}

\section{Conclusions}

The paper has discussed a new system for efficiently localizing a sound source, where a mobile robot is employedto enhance quality of data collection in a acoustic sensor network. That is, robot movements enable the system to gather more information of sound in environment as needed, which can then be fused with those measured by the static microphones to better locate a source. More specifically, all the sensors and 
the robot can synchronously communicate with each other and transmit the measurements to a central station through a pre-defined network. The recorded data is analysed by the SRP-PHAT algorithm to map a location of the sound source. Evaluation of the proposed system conducted in the realistic experiments has shown its effectiveness, promising for reallife applications.

\section{ACKNOWLEDGMENT}

This is an outcome from the research projects funded by the Blue Sky Scheme at University of Technology Sydney, Australia. We would thank Md Nurul Hoque for the assistance in the experiments.

\section{REFERENCES}

[1] H. Wang and P. Chu, "Voice source localization for automatic camera pointing system in videoconferencing," in Proc. EEE International Conference on Acoustics, Speech, and Signal Processing, Munich, Germany, August 1997, pp. 187-190.

[2] Y. Huang, J. Benesty, and G. W. Elko, "Passive acoustic source localization for video camera steering," in Proc. IEEE International Conference on Acoustics, Speech, and Signal Processing, Istanbul, Turkey, June 2000, pp. 909-912.

[3] F. Antonacci, M. Matteucci, D. Migliore, D. Riva, A. Sarti, M. Tagliasacchi, and S. Tubaro, "Tracking multiple acoustic sources in reverberant environments using regularized particle filter," in Proc. IEEE International Conference on Digital Signal Processing,, Cardiff, UK, July 2007, pp. 99-102.

[4] Q. Yan, J. Chen, G. Ottoy, and L. D. Strycker, "Robust AOA based acoustic source localization method with unreliable measurements," Signal Processing, vol. 152, pp. 13-21, 2018.

[5] M. I. Mandel, R. J. Weiss, and D. P. Ellis, "Model-based expectationmaximization source separation and localization," IEEE Transactions on Audio, Speech, and Language Processing, vol. 18(2), p. 382394, 2010.

[6] L. Parker, B. Birch, and C. Reardon, "Indoor target intercept using an acoustic sensor network and dual wavefront path planning," in Proc. IEEE/RSJ International Conferece on Intelligent Robots and Systems, Las Vegas, NV, USA, December 2003, pp. 278-283.

[7] K. Na, Y. Kim, and H. Cha, Acoustic sensor network-based parking lot surveillance system. Berlin, Heidelberg: Springer Berlin Heidelberg, 2009, p. 247262.

[8] L. Nguyen, J. VallsMiro, and X. Qiu, "Multilevel b-splines based learning approach for sound source localization," IEEE Sensors Journal, vol. Early Access, pp. 1-11, 2019.

[9] T. Latif, E. Whitmire, T. Novak, and A. Bozkurt, "Sound localization sensors for search and rescue biobots," IEEE Sensors Journal, vol. 16(10), pp. 3444-3453, 2016.

[10] L. Wang and A. Cavallaro, "Acoustic sensing from a multi-rotor drone," IEEE Sensors Journal, vol. 18(11), pp. 4570-4582, 2018.

[11] D. Su, K. Nakamura, K. Nakadai, and J. V. Miro, "Robust sound source mapping using three-layered selective audio rays for mobile robots," in Proc. IEEE/RSJ International Conferece on Intelligent Robots and Systems, Daejeon, Korea, October 2016, pp. 2771-2777.

[12] D. Su, T. V. Calleja, and J. V. Miro, "Towards real-time 3D sound sources mapping with linear microphone arrays," in Proc. IEEE International Conferece on Robotics and Automation, Singapore, Singapore, May 2017, pp. 1662-1668.

[13] M. Cobos, A. Marti, and J. J. Lopez, "A modified SRP-PHAT functional for robust real-time sound source localization with scalable spatial sampling," IEEE Signal Processing Letters, vol. 18(1), pp. 7174, 2011.

[14] A. Canclini, F. Antonacci, A. Sarti, and S. Tubaro, "Acoustic source localization with distributed asynchronous microphone networks," IEEE/ACM Transactions on Audio, Speech, and Language Processing, vol. 21(2), p. 439443, 2013.

[15] T. Dvorkind and S. Gannot, "Time difference of arrival estimation of speech source in a noisy and reverberant environment," Signal Processing, vol. 85(1), pp. 177-204, 2005.
[16] J. H. DiBiase, H. F. Silverman, and M. S. Brandstein, Robust Localization in Reverberant Rooms. Springer, Berlin, Heidelberg, 2001, ch. 8 , pp. $157-180$.

[17] K. Ho, "Bias reduction for an explicit solution of source localization using tdoa," IEEE Transactions on Signal Processing, vol. 60(5), p. 21012114, 2012.

[18] A. Canclini, P. Bestagini, F. Antonacci, M. Compagnoni, A. Sarti, and S. Tubaro, "A robust and low-complexity source localization algorithm for asynchronous distributed microphone networks," IEEE/ACM Transactions on Audio, Speech, and Language Processing, vol. 23(10), pp. 1563-1575, 2015.

[19] R. Parisi, A. Cirillo, M. Panella, and A. Uncini, "Source localization in reverberant environments by consistent peak selection," in Proc. IEEE International Conference on Acoustics, Speech, and Signal Processing, Honolulu, HI, USA, April 2007, pp. 37-40.

[20] M. Compagnoni, P. Bestagini, F. Antonacci, A. Sarti, and S. Tubaro, "Localization of acoustic sources through the fitting of propagation cones using multiple independent arrays," IEEE Transactions on Acoustics, Speech, and Signal Processing, vol. 20(7), pp. 1964-1975, 2012.

[21] L. Nguyen, S. Kodagoda, R. Ranasinghe, and G. Dissanayake, "Simulated annealing based approach for near-optimal sensor selection in gaussian processes," in Proc. IEEE International Conference on Control, Automation and Information Science, Ho Chi Minh city, Vietnam, November 2012, pp. 142-147.

[22] L. Nguyen, R. Ranasinghe, S. Kodagoda, and G. Dissanayake, "Sensor selection based routing for monitoring gaussian processes modeled spatial phenomena," in Proc. Australian Conference on Robotics and Automation, Wellington, New Zealand, December 2012.

[23] L. Nguyen, S. Kodagoda, R. Ranasinghe, and G. Dissanayake, "Locational optimization based sensor placement for monitoring gaussian processes modeled spatial phenomena," in Proc. 8th IEEE International Conference on Industrial Electronics and Applications, Melbourne, Australia, June 2013, pp. 1706-1711.

[24] _ - "Mobile robotic wireless sensor networks for efficient spatial prediction," in Proc. 2014 IEEE/RSJ International Conference on Intelligent Robots and Systems, Chicago, IL, USA, September 2014, pp. 1176-1181.

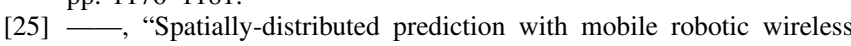
sensor networks," in Proc. 2014 13th IEEE International Conference on Control, Automation, Robotics and Vision, Marina Bay Sands, Singapore, December 2014, pp. 1153-1158.

[26] L. Nguyen, S. Kodagoda, and R. Ranasinghe, "Spatial sensor selection via Gaussian Markov random fields," IEEE Transaction on Systems, Man, and Cybernetics: Systems, vol. 46(9), pp. 1226-1239, 2016.

[27] L. Nguyen, G. Hu, and C. J. Spanos, "Spatio-temporal environmenta monitoring for smart buildings," in Proc. IEEE International Conference on Control and Automation, Ohrid, Macedonia, July 2017, pp. $277-282$.

[28] —_ "Sefficient spatio-temporal sensor deployments: A smart building application," in Proc. IEEE International Conference on Control and Automation, Ohrid, Macedonia, July 2017, pp. 612-617.

[29] L. Nguyen, S. Kodagoda, R. Ranasinghe, and G. Dissanayake, "Adaptive placement for mobile sensors in spatial prediction under locational errors," IEEE Sensors Journal, vol. 17(3), pp. 794-802, 2017.

[30] L. Nguyen, N. Ulapane, and J. VallsMiro, "Adaptive sampling for spatial prediction in environmental monitoring using wireless sensor networks: A review," in Proc. IEEE International Conference on Industrial Electronics and Applications, Wuhan, China, May 2018, pp. 346-351.

[31] L. Nguyen, G. Hu, and C. J. Spanos, "Efficient sensor deployments for spatio-temporal environmental monitoring," IEEE Transaction on Systems, Man, and Cybernetics: Systems, vol. Early Access, pp. 1-11, 2018.

[32] L. Nguyen, S. Kodagoda, R. Ranasinghe, and G. Dissanayake, "Information-driven adaptive sampling strategy for mobile robotic wireless sensor network," IEEE Transaction on Control Systems Technology, vol. 24(1), pp. 372-379, 2016.

[33] J. Chen, J. Benesty, and Y. Huang, "Time delay estimation in room acoustic environments: An overview," EURASIP Journal on Advances in Signal Processing, vol. 2006, pp. 1-19, 2006.

[34] C. H. Knapp and G. C. Carter, "The generalized correlation method for estimation of time delay," IEEE Transactions on Acoustics, Speech, and Signal Processing, vol. 24(4), pp. 320-327, 1976.

[35] [Online]. Available: https://fetchrobotics.com/ 